Article

\title{
The Circadian Effect Versus Mesopic Vision Effect in Road Lighting Applications
}

\author{
Min Li ${ }^{1}$, Peiyu $\mathrm{Wu}^{2}$, Jianhua Ding ${ }^{2,3}$, Qi Yao ${ }^{2, *}$ (1) and Jiaqi Ju ${ }^{4, *}$ \\ 1 Institute for Electric Light Sources, Fudan University, Shanghai 200433, China; lm_ss@fudan.edu.cn \\ 2 College of Architecture and Urban Planning, Shenzhen University, Shenzhen, Guangdong 518060, China; \\ 1900321007@email.szu.edu.cn (P.W.); jianhua_ding@szu.edu.cn (J.D.) \\ 3 School of Architecture, Northeastern University, Liaoning 110004, China \\ 4 School of Science, Shanghai Institute of Technology, Shanghai 201418, China \\ * Correspondence: yaoqi@szu.edu.cn (Q.Y.); jujiaqi@sit.edu.cn (J.J.)
}

Received: 11 August 2020; Accepted: 29 September 2020; Published: 6 October 2020

check for updates

Featured Application: This work clarifies light sources' circadian effect and mesopic vision effect in road lighting for the first time, and guides circadian and efficient outdoor lighting application.

\begin{abstract}
Several models on the circadian effect have been applied to indoor circadian lighting design, but applications in road lighting have not yet been clarified. Based on existing models and circadian research, we examined equivalent melanopic lux (EML), circadian light $\left(\mathrm{CL}_{\mathrm{A}}\right)$, and circadian stimulus (CS) representing the circadian effect and the S/P ratio representing the mesopic vision effect, among a dataset of light sources at photopic adaptation illuminance values of 1,3,10,30, and $1001 x$. The results show that the $\mathrm{S} / \mathrm{P}$ ratio correlates with EML and CS (or $\mathrm{CL}_{\mathrm{A}}$ ) much stronger than it correlates with color temperature. The EMLs of light sources are below 50 EML in mesopic vision, and the CSs of most light sources are below or around the threshold value of 0.05 . We conclude that the circadian effect is not a significant issue in mesopic vision under most conditions and that optimization for mesopic efficiency is still a good strategy. There are quite a few light sources that may achieve both ideal mesopic efficiency and low CS. This work clarifies the circadian effect and mesopic vision effect performance of light sources in mesopic vision and will help guide choosing suitable light sources and optimization strategies for road lighting.
\end{abstract}

Keywords: S/P ratio; EML; CS; CCT; mesopic vision; photopic vision

\section{Introduction}

There are two kinds of visual imaging photoreceptors [1], cone and rod cells, and one kind of non-visual imaging photoreceptor, ipRGCs [2,3]. The cones, rods, and ipRGCs relate to the spectral sensitivity of photopic vision, scotopic vision, and melatonin suppression $[4,5]$, respectively. For the visual imaging system, the activation levels of the cones and rods differ at different luminance (or illuminance) ranges. Road lighting is mainly in the mesopic vision stage, where both cones and rods activate. For non-visual imaging systems, melatonin suppression occurs under a large range of illuminance levels, even as low as 1 lx [6]. Most studies focus on indoor circadian lighting applications with illuminance values of about 100 to $1000 \mathrm{~lx}$ in photopic vision. Only a few works [7] focused on dim outdoor lighting applications (<30 lx), which might also be in the mesopic vision stage. It is not clear how the circadian effect and mesopic vision effect work for dim road lighting applications.

For road lighting applications, the lighting should meet several needs, including the detection of small objects and safety [8,9]. Generally, luminance for road lighting is about 0.5 to $2 \mathrm{~cd} / \mathrm{m}^{2}$ for 
energy-saving concerns in a mesopic vision state. Mesopic vision is a vision state in which both cone and rod cells activate at a luminance level from 0.005 to $5 \mathrm{~cd} / \mathrm{m}^{2}$ [10]. In road lighting, the empirical rule of $\mathrm{E}=\mathrm{L} \pi / \rho$ governs transforming luminance to illuminance, where $\mathrm{E}$ is the illuminance, $\mathrm{L}$ is the luminance, and $\rho$ is the reflectance rate of the road. Roads are mainly paved with two materials, concretes and asphalts. There are several kinds of common concrete road surface materials. The average reflectance rate of concrete's surface is about 0.18 to 0.32 [11], according to data from the National Aeronautics and Space Administration (NASA) Jet Propulsion Laboratory's Advanced Spaceborne Thermal Emission Reflection Radiometer (ASTER) library. The average reflectance rate of the asphalt surface varies greatly from several percent to about 70 percent due to the aging process [12]. We adopt a reflectance rate $\rho$ as 0.3 for the road surface. For road lighting luminance measurements, one method is to measure from $83 \mathrm{~m}$ ahead of a vehicle at a 1.5-meter height with a 1-degree angle representing the driver's view. Here, we adopt the above-mentioned empirical formula to calculate road luminance more simply. Therefore, the illuminance levels for mesopic vision can be roughly taken as 0.05 to $50 \mathrm{~lx}$. As is well known, human sensitivity to light shifts to shorter wavelengths from photopic to scotopic vision [13]. The peak wavelengths of photopic and scotopic spectra sensitivity are 555 and $507 \mathrm{~nm}$, respectively. A mesopic vision state is a combination of these two vision states, and the lower the luminance level is, the greater the sensitivity to blue light. Light sources with rich short wavelengths may have high mesopic efficiency. This was a claimed advantage during the transition process from high-pressure sodium to white light-emitting diode (LED) light sources a decade ago. Many works have been dedicated to optimizing high mesopic efficiency light sources [14-16]. Although mesopic vision applications in road lighting have been widely accepted, Gibbson et al.'s work showed that there are no significant differences in the detection distances for high and low correlated color temperature (CCT) sources when the speed is over $56 \mathrm{~km} / \mathrm{h}$ [17]. This might change the optimization strategy under certain conditions.

The circadian rhythm is mainly influenced by distinct photoreceptors in the eye, melanopsin-containing intrinsically photosensitive retinal ganglion cells (ipRGCs) [2,3]. The spectral sensitivity of melanopsin is similarly invariant across species, with peak intensity at approximately $480 \mathrm{~nm}$ [18-22]. Melatonin secretion is decided by both intensity and spectrum. In almost all species, including human beings, melatonin levels are high at night and low during the day [23,24]. Human physiology and behavior are influenced by retinal illumination [25]. To better apply the circadian design mechanism, the circadian parameters should be better expressed, similar to illuminance. Therefore, researchers have proposed different metrics for this application. Lucas proposed equivalent melanopic lux (EML) [5] based on melatonin spectral sensitivity. In the nocturnal application, $50 \mathrm{EML}$ is taken as the upper limit [26]. However, the circadian rhythm may be influenced not only by ipRGCs but also by rods and cones. Thus, Rea proposed a comprehensive model expressed as circadian light $\left(\mathrm{CL}_{\mathrm{A}}\right)$ and circadian stimulus $(\mathrm{CS})$ to describe the circadian impact considering influences from all neuro paths $[27,28]$. The CS ranges from 0 to 0.7 , namely, from 0 to $70 \%$ melatonin suppression levels. In the daytime, the CS $\geq 0.3$ (equivalent to $30 \%$ melatonin suppression) is required for at least $1 \mathrm{~h}$ to maintain circadian health [29]. Acosta adopted a CS equal to 0.1 as the threshold [30], and Rea et al. used a CS value of 0.05 as the target threshold [28]. A series of indoor circadian lighting research [31,32] and optimization works $[33,34]$ show that circadian-guided lighting design helps improve light quality for human health. This influence is significant in a brightly lit environment. However, some research also shows that there is likewise significant melatonin suppression under dim light conditions [35]. Wright et al. showed that illuminance as low as $1.5 \mathrm{~lx}$ affects circadian rhythms [36]. Under certain conditions, as little as 1 lx or less can suppress melatonin in humans [30]. Andrew et al. [37] showed that a 50\% melatonin suppression level corresponds to $13.5 \mathrm{~lx}$ for Hour 1, $19.4 \mathrm{~lx}$ for Hour 2, and $38.9 \mathrm{~lx}$ for Hour 3. These illuminances are mainly in the mesopic vision range.

Exposure time under light is also an important factor that decides the melanopic effect. Most studies adopt 'hour' as their measurement scale [27,28,31,37], and a one-hour duration is used in most studies. If the exposure time is much shorter than one hour, the melanopic effect might be minimized. 
Recent studies found that ipRGCs, as well as $\mathrm{S}$ cones, might enhance scene brightness in the photopic range covering low, moderate, and high illuminance levels (i.e., 2 to $201 x$ [38], 6.3 lx, 108 lx [39], and $1000 \mathrm{~lx}$ [40]). This might be a good measure, but the relevant mechanism study is currently still in progress. Moreover, mesopic models might already include scene brightness enhancement in the mesopic luminance range, although former researchers might not think that this phenomenon is caused by ipRGC. We thus do not include this part in the research.

The spectral sensitivity of mesopic vision peaks at $507 \mathrm{~nm}$ and melanopsin at $484 \mathrm{~nm}$ for crystalline lens transmittance. These two action spectra are close to each other and sensitive to blue light. At nighttime, rich blue light sources may improve mesopic efficiency and suppress melatonin secretion. High correlated color temperature (CCT) white LEDs suppress melatonin secretion about 4 to 5 times [41] more strongly than high-pressure sodium (HPS) light sources in humans using a fitted action spectrum of melatonin suppression [42]. Meanwhile, white LEDs may provide mesopic efficiency 2 times higher than HPS [15]. The relation or tradeoff between mesopic efficiency and the circadian (or melatonin suppression) effect requires clarity.

\section{Materials and Methods}

\subsection{Materials}

To test the circadian effect and mesopic efficiency under a dim road lighting environment, we calculated the $\mathrm{CCT}, \mathrm{S} / \mathrm{P}$ ratio, $\mathrm{EML}, \mathrm{CL}_{\mathrm{A}}$, and $\mathrm{CS}$ of a large set light source and analyzed their relationships. We adopted the light source data from IES TM30-15 for 318 light sources [43], as shown in Figure 1, including all kinds of light sources, such as incandescent lamps, fluorescent lamps, high-pressure intensity discharge lamps, and LEDs, to assess EML, $\mathrm{CL}_{\mathrm{A}}$, and CS in mesopic and near photopic vision. We set the photopic adaptation illuminance levels at 1, 3, 10, 30, and $100 \mathrm{~lx}$. These illuminance levels are equal to $0.1,0.3,1,3$, and $10 \mathrm{~cd} / \mathrm{m}^{2}$ for asphalt or concrete roads with a reflectance rate equal to 0.3 , covering both mesopic and near photopic vision luminance ranges. In the assessment of the melanopic effect, retinal illuminance is required. For simplicity, we assume that retinal illuminance is equal to mesopic illuminance in mesopic vision and photopic adaptation illuminance in photopic vision.

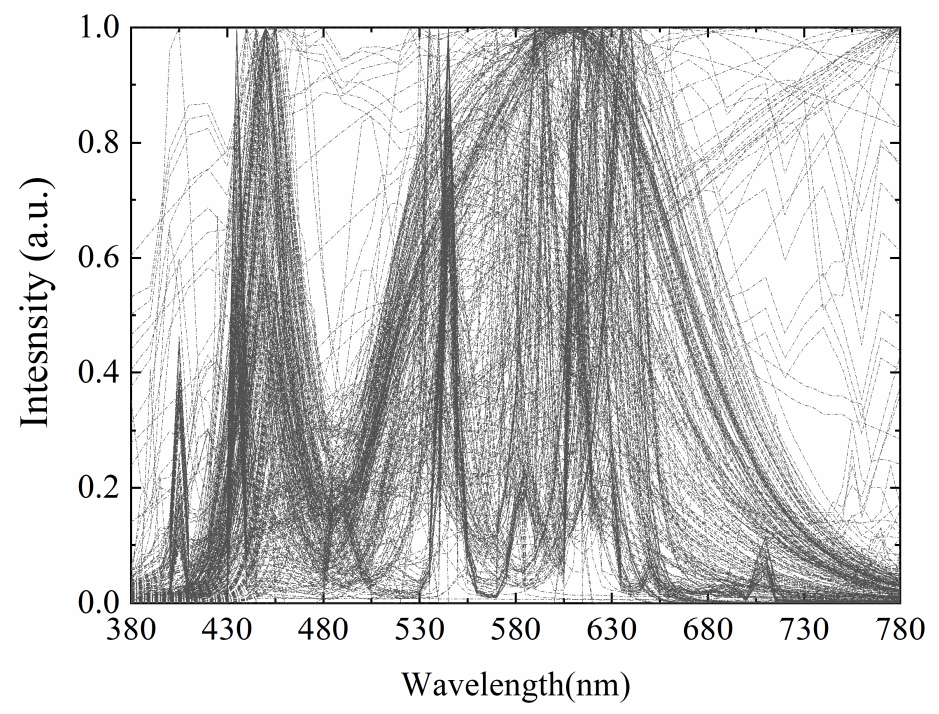

Figure 1. Spectral power distributions of light sources.

Figure 2a shows the spectral sensitivity curve of the photopic vision $\mathrm{V}_{\lambda}$, scotopic vision $\mathrm{V}_{\lambda}{ }_{\lambda}, \mathrm{S}$-con fundamental $S_{\lambda}$, and melanopsin corrected with the lens, which are used in the calculations. We further 
adopt typical road lighting sources, an HPS light source, and a low CCT phosphor-converted (PC) LED and high CCT PC LED, as shown in Figure $2 b$, to test their circadian and mesopic performance.

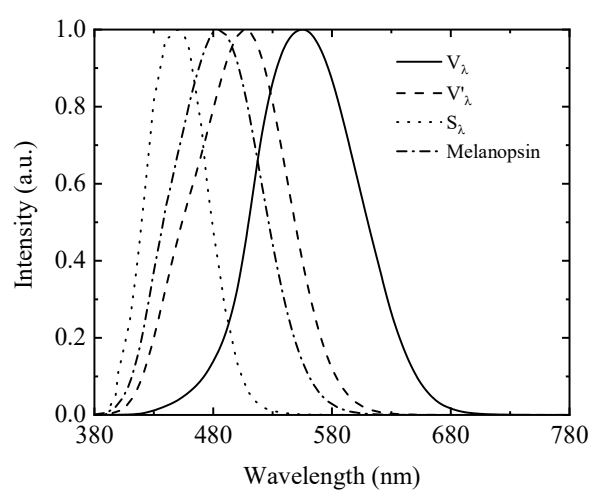

(a)

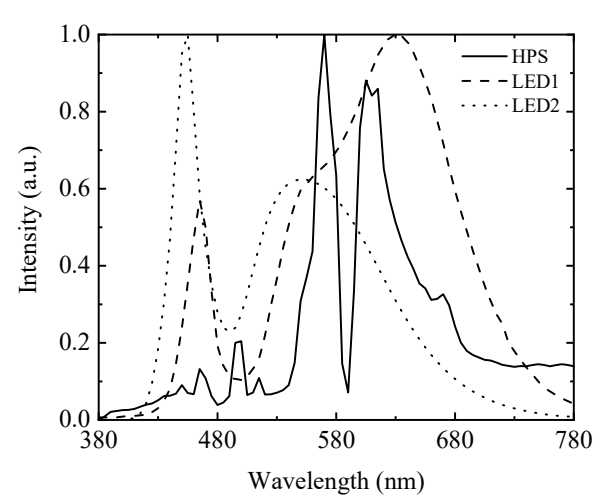

(b)

Figure 2. (a) The relative spectral sensitivity curve of the photopic vision $\mathrm{V}_{\lambda}$, scotopic vision $\mathrm{V}^{\prime}{ }_{\lambda}$, $\mathrm{S}$-cone fundamental $S_{\lambda}$, and melanopsin sensitivity curve $\mathrm{Mc}_{\lambda}$ (corrected for crystalline lens transmittance). (b) Spectral power distributions of high-pressure sodium (HPS), phosphor-converted (PC) light-emitting diode (LED) 1, and PC LED 2 with CCT 2238 K, 2497 K, and 6060 K, respectively.

\section{2. $S / P$ Ratio}

The $\mathrm{S} / \mathrm{P}$ ratio is a calculation of the scotopic lumens of a lamp divided by the photopic lumens. The calculation formula is shown in Equation (1), where $E_{\lambda}$ represents the light-source's spectral power distribution, $K_{m}$ is the maximum photopic luminous efficacy $\left(=683 \mathrm{~lm} / \mathrm{W}\right.$ at $555 \mathrm{~nm}$ ), and $K_{m}{ }^{\prime}$ is the maximum scotopic luminous efficacy $(=1700 \mathrm{~lm} / \mathrm{W}$ at $507 \mathrm{~nm})$ :

$$
S / P \text { ratio }=\frac{K_{m}^{\prime} \int_{380}^{780} E_{\lambda} V_{\lambda} d \lambda}{K_{m} \int_{380}^{780} E_{\lambda} V_{\lambda} d \lambda}
$$

\subsection{Equivalent Melanopic Lux}

EML is a metric for measuring the biological effects of light on humans and is defined as shown in Equation (2) [5].

$$
E M L=72983.25 \int E_{\lambda} M c_{\lambda} d \lambda
$$

\subsection{Circadian Light and Circadian Stimulus}

$\mathrm{CL}_{\mathrm{A}}$ mathematically describes the modeled spectral sensitivity of the human circadian system by Rea et al. [28] and is defined as shown in Equation (3), where $C L_{A}$ is the circadian light, 1548 is the constant setting for the normalization of $C L_{A}$ such that $2856 \mathrm{~K}$ blackbody radiation at $1000 \mathrm{~lx}$ has a $C L_{A}$ value of $1000, E_{\lambda}$ is the spectral power distribution, $S_{\lambda}$ is the S-cone fundamental, $m p_{\lambda}$ is the macular pigment transmittance, RodSat is the half-saturation constant for bleaching rods $=6.5 \mathrm{~W} / \mathrm{m}^{2}$, $k=0.2616, a_{b-y}=0.7000$, and $a_{r o d}=3.3000 . \mathrm{CL}_{\mathrm{A}}$ is measured in units of spectrally weighted flux per unit area. $C S$ is a proposed metric based on the $\mathrm{CL}_{\mathrm{A}}$ value with a maximum value of 0.7 , as shown in Equation (4) [28], and is dimensionless. Due to a blue versus yellow (b-y) mechanism [28], the $\mathrm{CL}_{\mathrm{A}}$ is expressed by a piecewise function depending on whether b-y, namely $\int \frac{S_{\lambda}}{m p_{\lambda}} E_{\lambda} d \lambda-k \int \frac{V_{\lambda}}{m p_{\lambda}} E_{\lambda} d \lambda,>0$ or $<0$. If the yellow stimuli dominate, only the intrinsic sensitivity of the ipRGC contributes to the 
circadian response; otherwise, all photoreceptors contribute to the circadian response. The default calculations of $C L_{A}$ and $C S$ correspond to a one-hour duration of exposure.

$$
\begin{aligned}
& C L_{A}=\left\{\begin{array}{cc}
1548\left[\int M c_{\lambda} E_{\lambda} d \lambda+\left(a_{b-y}\left(\int \frac{S_{\lambda}}{m p_{\lambda}} E_{\lambda} d \lambda-k \int \frac{V_{\lambda}}{m p_{\lambda}} E_{\lambda} d \lambda\right)-a_{r o d}\left(1-e \frac{-\int V r_{\lambda} E_{\lambda} d \lambda}{\operatorname{RodSat}}\right)\right)\right] \\
1548 \int M c_{\lambda} E_{\lambda} d \lambda \quad & \text { if } \int \frac{S_{\lambda}}{m p_{\lambda}} E_{\lambda} d \lambda-k \int \frac{V_{\lambda}}{m p_{\lambda}} E_{\lambda} d \lambda>0 \\
\text { if } \int \frac{S_{\lambda}}{m p_{\lambda}} E_{\lambda} d \lambda-k \int \frac{V_{\lambda}}{m p_{\lambda}} E_{\lambda} d \lambda \leq 0
\end{array}\right. \\
& C S=0.7-0.7 /\left[1+\left(\frac{C L_{A}}{355.7}\right)^{1.1026}\right]
\end{aligned}
$$

\subsection{Analysis Method}

We conducted a Shapiro-Wilk normality test of the data and a Spearman correlation analysis between the independent variables (CCT and S/P ratio) and dependent variables (namely EML and CS). We also conducted a curve fitting analysis of the corresponding variables.

\section{Results}

\subsection{EML as a Function of the CCT and S/P Ratio}

Figure 3 shows EML as functions of the CCT and S/P ratio at a $11 x$ level. The EML is linear to the illuminance value, so the EML values at different illuminance levels can be achieved by multiplying by an intensity factor $\mathrm{k}$ of $3,10,30$, and 100. The goodness of fit for EML and CCT reaches a moderate level of 0.685 , while the EML and S/P ratio reaches an extremely high level of 0.989 . These data do not conform to the normal distribution at a 0.05 significant level. We thus use a Spearman correlation analysis. The Spearman correlation coefficients between EML and CCT and between EML and $\mathrm{S} / \mathrm{P}$ ratio are about 0.77 and 0.99 , respectively.

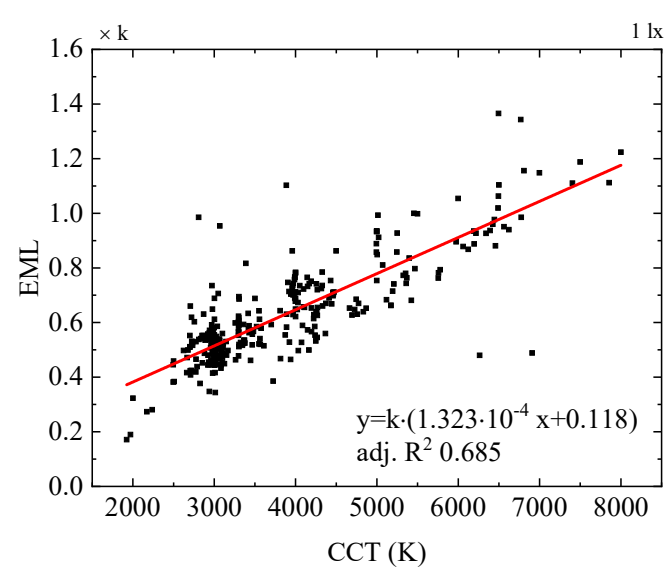

(a)

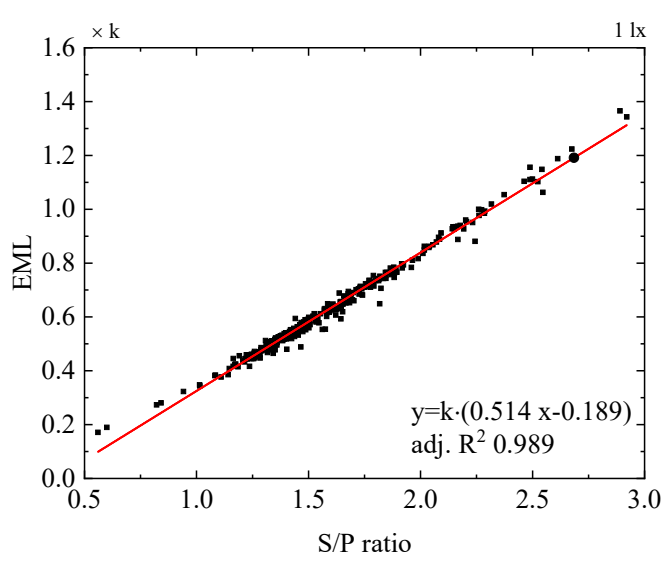

(b)

Figure 3. (a) Equivalent melanopic lux (EML) plot against correlated color temperature (CCT) at an illuminance value of $1 \mathrm{~lx}$. In the graph, $x$ represents CCT, y represents EML, and $\mathrm{k}$ is the adjusting coefficient equal to the illuminance level, e.g., 1 at a 1 lx level. (b) EML plot against the S/P ratio at an illuminance of $1 \mathrm{~lx}$.

\section{2. $C L_{A}$ and $C S$ as a Function of the $C C T$ and $S / P$ Ratio}

Figures 4 and 5 show $\mathrm{CL}_{\mathrm{A}}$ and $\mathrm{CS}$ as functions of the CCT and S/P ratio, respectively, at 1, 3, 10, 30, and $100 \mathrm{~lx}$. At each illuminance level, both $\mathrm{CL}_{\mathrm{A}}$ and $\mathrm{CS}$ increase with the CCT and S/P ratio. However, the $\mathrm{CL}_{\mathrm{A}}$ and $\mathrm{CS}$ scatters are divided into two clusters. Both $\mathrm{CL}_{\mathrm{A}}$ and $C S$ increase with illuminance levels. However, despite presetting a similar trend, they are not simple linear relations. 


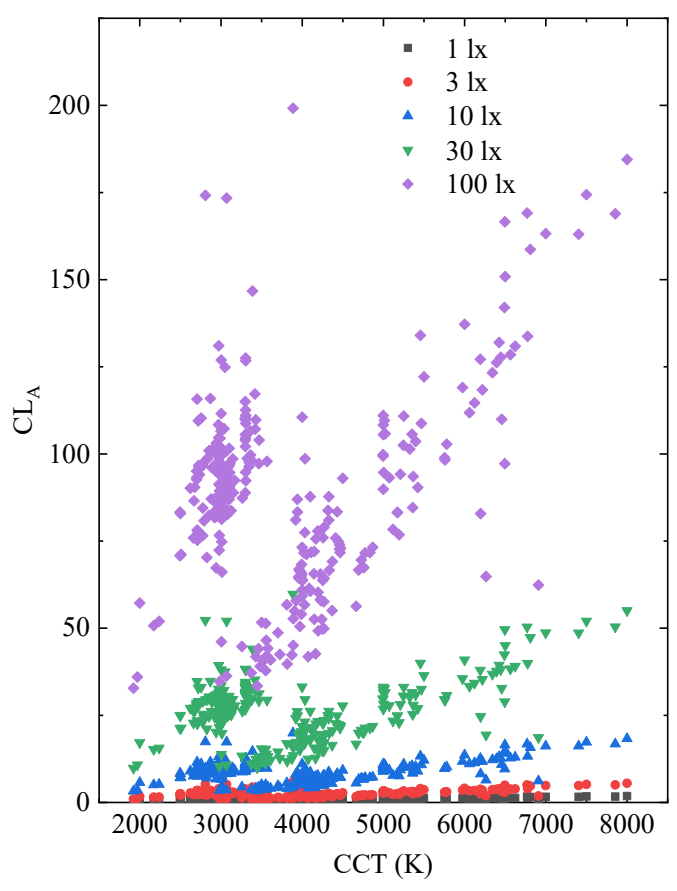

(a)

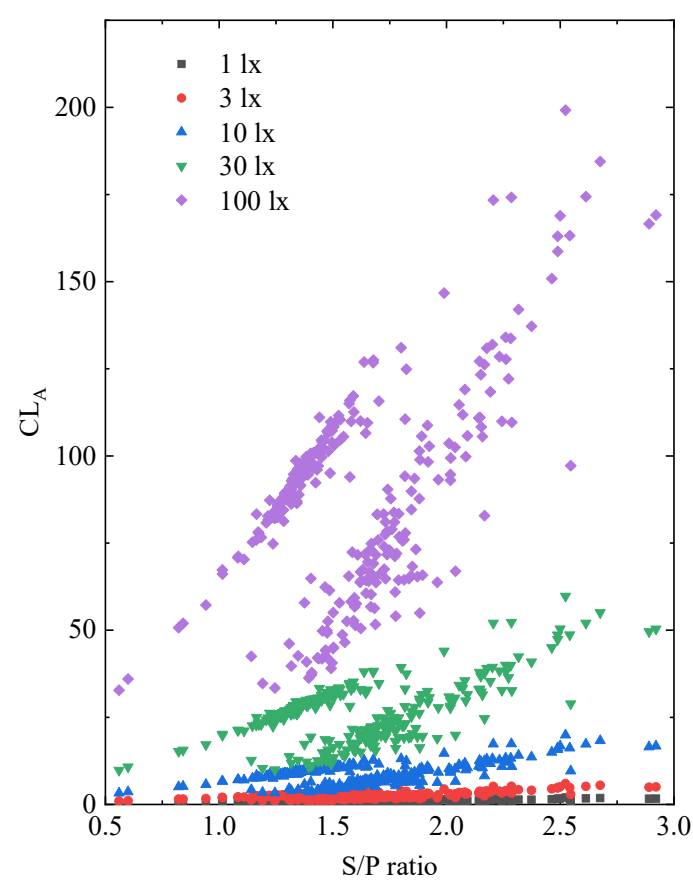

(b)

Figure 4. Circadian light $\left(\mathrm{CL}_{\mathrm{A}}\right)$ plot against the (a) $\mathrm{CCT}$ and $(\mathbf{b}) \mathrm{S} / \mathrm{P}$ ratio.

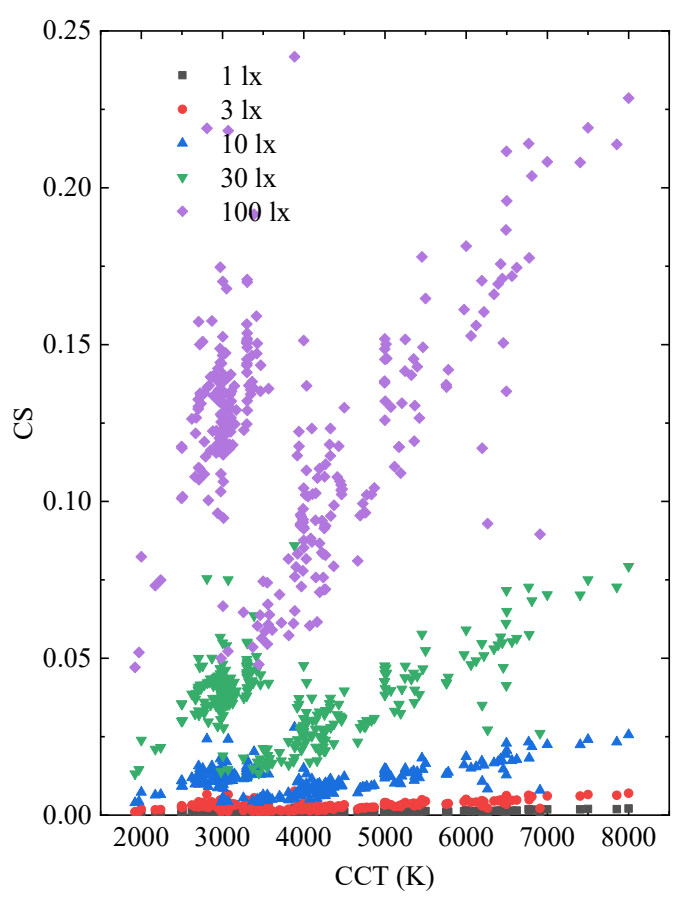

(a)

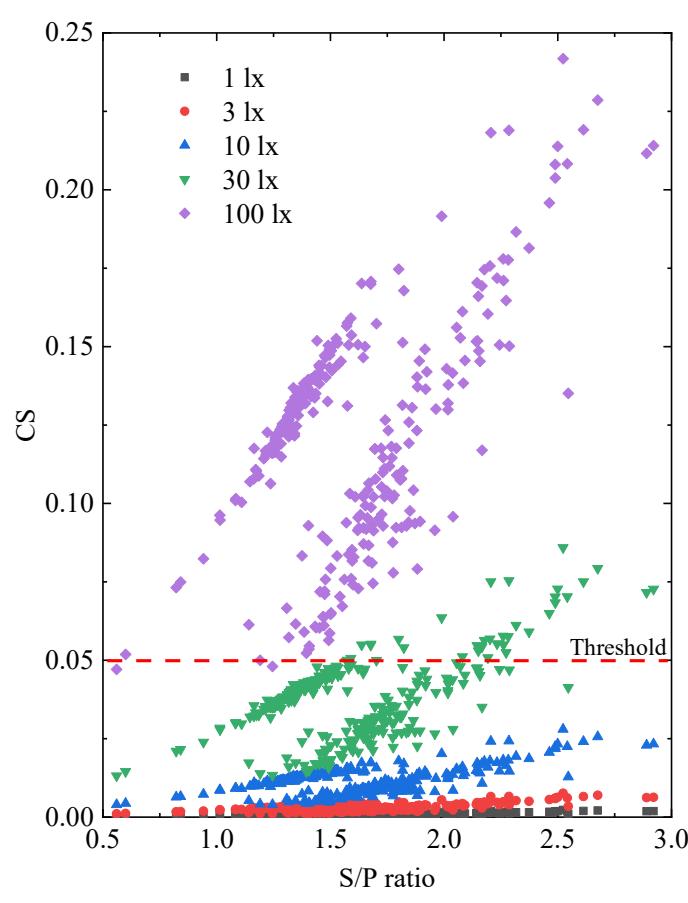

(b)

Figure 5. Circadian stimulus (CS) plot against the (a) CCT and (b) S/P ratio.

Table 1 shows the EML, CL , and CS of HPS, with low CCT LED and high CCT LED at various photopic adaptation illuminance levels. HPS, with a minimum CCT of $2238 \mathrm{~K}$ and an S/P ratio equal to $0.8417<1$, shows a minimum EML, $\mathrm{CL}_{\mathrm{A}}$, and CS. The low CCT LED shows a moderate level of EML, 
$\mathrm{CL}_{\mathrm{A}}$, and $\mathrm{CS}$, which are about 1.5 times that of HPS. The high CCT LED has a maximum EML, $\mathrm{CL}_{\mathrm{A}}$, and CS, which are about 2 to 3 times the values of HPS.

Table 1. The EML, $\mathrm{CL}_{\mathrm{A}}$, and CS of HPS and the low CCT LED and high CCT LED at different photopic adaptation illuminance levels.

\begin{tabular}{|c|c|c|c|c|}
\hline & & HPS & LED 1 & LED 2 \\
\hline \multicolumn{2}{|r|}{ CCT (K) } & 2238 & 2497 & 6060 \\
\hline \multicolumn{2}{|r|}{$\mathrm{S} / \mathrm{P}$ ratio } & 0.8417 & 1.1642 & 2.0711 \\
\hline \multirow{5}{*}{ EML } & $1 \mathrm{~lx}$ & 0.2807 & 0.4453 & 0.8781 \\
\hline & $31 x$ & 0.8421 & 1.3358 & 2.6342 \\
\hline & $10 \mathrm{~lx}$ & 2.8069 & 4.4527 & 8.7808 \\
\hline & $30 \mathrm{~lx}$ & 8.4206 & 13.3580 & 26.3424 \\
\hline & $100 \mathrm{~lx}$ & 28.0687 & 44.5265 & 87.8080 \\
\hline \multirow{5}{*}{$\mathrm{CL}_{\mathrm{A}}$} & $1 \mathrm{~lx}$ & 0.5196 & 0.8330 & 1.1096 \\
\hline & $3 \mathrm{~lx}$ & 1.5588 & 2.4989 & 3.3293 \\
\hline & $10 \mathrm{~lx}$ & 5.1959 & 8.3298 & 11.1040 \\
\hline & $30 \mathrm{~lx}$ & 15.5878 & 24.9893 & 33.3659 \\
\hline & $100 \mathrm{~lx}$ & 51.9594 & 83.2978 & 111.8434 \\
\hline \multirow{5}{*}{ CS } & $1 \mathrm{~lx}$ & 0.0005 & 0.0009 & 0.0012 \\
\hline & $3 \mathrm{~lx}$ & 0.0018 & 0.0029 & 0.0040 \\
\hline & $10 \mathrm{~lx}$ & 0.0066 & 0.0110 & 0.0150 \\
\hline & $30 \mathrm{~lx}$ & 0.0216 & 0.0355 & 0.0480 \\
\hline & $100 \mathrm{~lx}$ & 0.0750 & 0.1175 & 0.1528 \\
\hline
\end{tabular}

\section{Discussion}

The circadian effect is a non-visual imaging effect, mainly caused by a third photoreceptor. The two types of visual photoreceptors, cones and rods, which are sensitive to luminance and color, also play important roles in circadian responses under specific situations. EML takes the melanopic sensitivity curve as the main influence of the circadian response, while $\mathrm{CL}_{\mathrm{A}}(\mathrm{CS})$ is a comprehensive model dedicated to measuring the general circadian response.

EML includes the melanopic sensitivity curve, which is close to the scotopic sensitivity curve. There is moderate correlation between EML and CCT and extremely high correlation between the EML and S/P ratio due to the similarity of the melanopic and scotopic sensitivity curves. It is practical to predict the EML value using the $\mathrm{S} / \mathrm{P}$ ratio in road lighting. EML only includes the melatonin sensitivity curve, which might adapt to an ideal situation for the circadian response. However, all photoreceptors contribute to the circadian response. Therefore, EML may not be practical for some situations. At present, the International Well Building Institute [26] recommends the use of EML for circadian lighting evaluations by setting a threshold in various scenes and requires no more than 50 EML under nocturnal lighting. Figure 6 shows EML as a function of the S/P ratio at a 30 lx level, where the maximum EML is below 50. Rea et al. [44] reported that two high CCT LEDs ( $\geq 5200 \mathrm{~K}$ ) might cause a small stimulating effect (namely, $12-15 \%$ nocturnal melatonin suppression) in the human circadian system after one hour of exposure at a $95 \mathrm{~lx}$ level under outdoor lighting, while low CCT light sources would not. These results are consistent with ours. Thus, in a mesopic vision state, the melanopic suppression level might not be a significant problem.

Because $C_{A}$ and $C S$ are piecewise functions, there are no significant correlations between the $\mathrm{CL}_{\mathrm{A}}$ (or CS) and $\mathrm{S} / \mathrm{P}$ ratio using the Spearman correlation test. We categorized these points into two groups according to their b-y relation. For example, at a $10 \mathrm{~lx}$ illuminance level, there is a high goodness-of-fit between the CS and S/P ratio of 0.955 for $b-y<0$ and 0.842 for $b-y>0$, as shown in Figure 7. However, in actual applications, this conclusion might be impractical due to the trouble of declaring the $b-y$ value. 


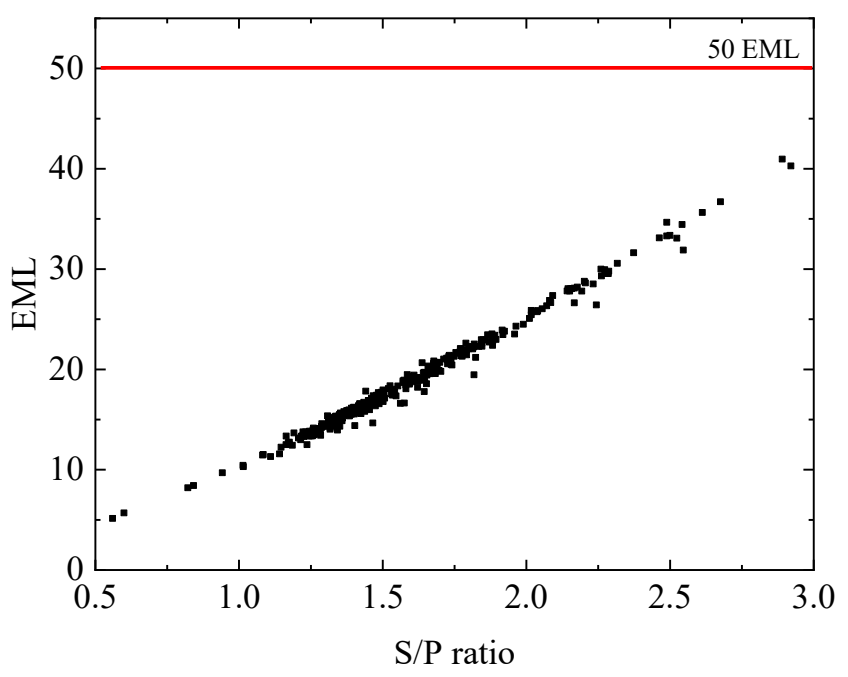

Figure 6. EML as a function of the $\mathrm{S} / \mathrm{P}$ ratio at $30 \mathrm{~lx}$.

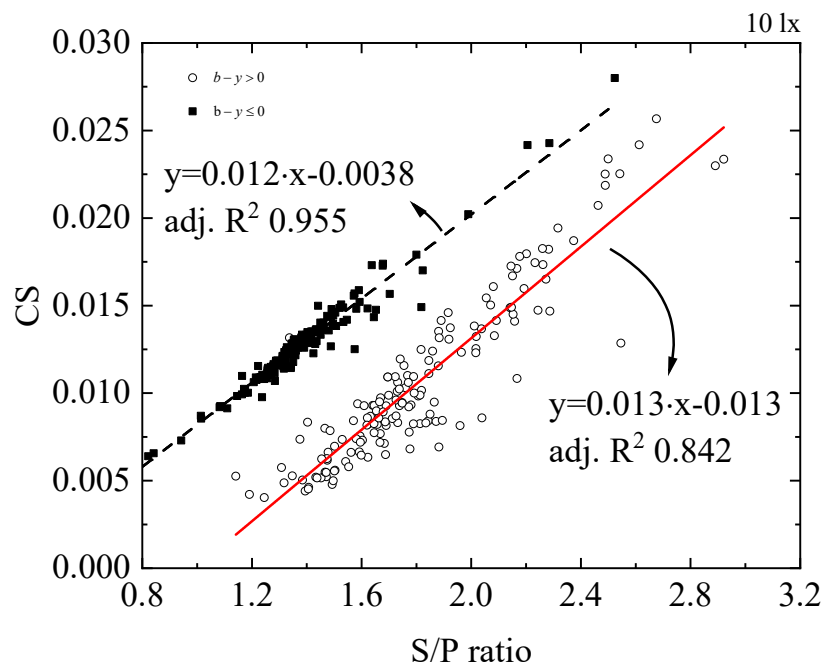

Figure 7. CS as a function of the S/P ratio at $10 \mathrm{~lx}$.

A high CCT light source is likely to have high EML and CS. However, there is a moderate correlation between EML and CCT and a weak correlation between CS and CCT. Moreover, due to the divergence of CS caused by the $b-y$ mechanism, at each varying CCT, there are great variations among the CS values. Therefore, CCT is not a good indicator for determining the circadian response levels.

In the mesopic vision range, intensity might reach the threshold of suppressing melatonin. Therefore, although blue light improves mesopic efficiency and suppresses melatonin, this might not be a significant issue according to present knowledge on circadian responses. Improving mesopic efficiency is still a good optimization strategy for road lighting. Previous work on optimizing light sources might still work in road lighting applications. However, some people are sensitive to light, and about 10 to $301 x$ may cause $50 \%$ melatonin suppression after about one-hour exposure [37]. Thus, choosing light sources with relatively high S/P ratios and moderate EML and CS would be a better strategy. In some roadway activities, such as walking and cycling activities, the duration of exposure to artificial light might be less than one hour, so there might be a smaller suppression effect. By taking the road surface reflection rate as 0.3 , we transformed photopic adaptation illuminance to mesopic illuminance for 318 light sources. Figure 8 shows the CS plot against mesopic illuminance at a photopic adaptation illuminance of $10 \mathrm{~lx}$. When mesopic illuminance increases from 10 to $11 \mathrm{~lx}$ (i.e., a 10\% improvement), although the CSs vary in a large range, there is still a group of light sources with very 
low CSs, as shown in the red dashed circle. It is possible to find ideal light sources with relatively high mesopic efficiency and low CS in application.

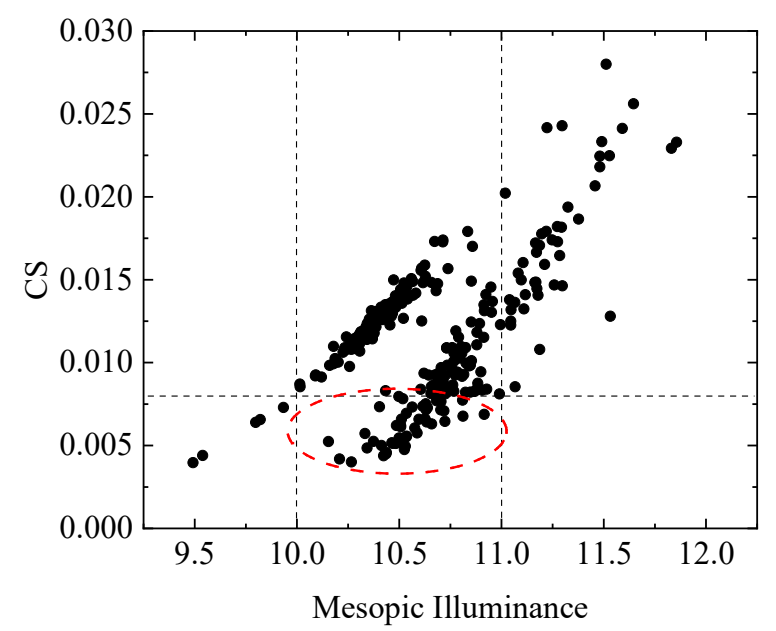

Figure 8. CS as a function of mesopic illuminance at $10 \mathrm{~lx}$. Within the red dashed circle, CSs stay at a relatively low level, but mesopic illuminance increases.

The mesopic vision design of road lighting work is dedicated to providing a good brightness lighting environment for pedestrians and drivers. In the mesopic vision state, peripheral vision, which contains many rod cells, plays an important role and works for most dim light situations. However, Gibbons et al. [17] noted that mesopic vision-designed light may not help improve visibility at night for high driving speeds. For relatively high driving speeds, foveal vision may be the dominant mode of detection. Therefore, for some road lighting that is used for people driving cars, rather than pedestrians or cyclists, road lighting strategy might not focus on mesopic vision design.

There are several situations in which road lighting luminance levels are suitable for photopic vision, and some of the results for EML, $\mathrm{CL}_{\mathrm{A}}$, and CCT may still work. Both EML and $\mathrm{CL}_{\mathrm{A}}$ (under the condition of $b-y<0$ ) are linear with illuminance levels, and their characteristics with varying CCTs are quite similar in photopic and mesopic vision. $\mathrm{CL}_{\mathrm{A}}$ under the condition $\mathrm{b}-\mathrm{y}>0$ does not have a simple linear relationship with intensity, but the trend along with CCT similarly remains. The low/high CCT PC LED is about 1.5/3 times higher than the HPS in EML, 1.5/2.3 times higher in CS, and $40 \% / 150 \%$ higher in the S/P ratio. These results are different from those of previous work [41] showing that high CCT LED suppresses melatonin secretion about 4 to 5 times more strongly than HPS. Therefore, in mesopic vision applications, we recommend using relatively high CCT LED (about 4000 to $5000 \mathrm{~K}$ ) light sources to achieve high mesopic efficiency because both the EML and CS of light sources are below or around the threshold; in photopic vision applications, we recommend using HPS or low CCT light sources (2000 to $4000 \mathrm{~K}$ ) in road lighting for low EML and CS levels. In a low-to-moderate photopic range, short-wavelength output enhances scene brightness [39,45], which might indicate a partial contribution of cones and ipRGCs. This brightness mechanism is still being studied and might affect the optimization strategy for light source SPD values in outdoor lighting applications. Although we did not include this scene brightness enhancement mechanism in the present work, it deserves attention and further study.

\section{Conclusions}

In this article, we focused on light sources' circadian effects and the mesopic vision effect in road lighting. Based on an examination of the EML, CS, and S/P ratio of over 300 light sources, we conclude that (1) the EMLs and CSs of most light sources in a mesopic vision state are below or around the melatonin suppression threshold according to present research; (2) most light sources can cause a very small circadian response in a mesopic vision state, which might influence a few people who are 
sensitive to light. Therefore, under most conditions, there is no need to consider the trade-off between the circadian effect and mesopic efficiency. Moreover, reaching high mesopic efficiency is a good optimization strategy under mesopic vision for road lighting. Further, the CCT cannot be used as an accurate indicator for determining the potential circadian effects of light sources.

Author Contributions: Conceptualization, M.L. and Q.Y.; methodology, Q.Y.; software, P.W.; validation, M.L., Q.Y., and J.D.; formal analysis, P.W., Q.Y., and J.J.; resources, Q.Y.; data curation, Q.Y.; writing-original draft preparation, M.L., Q.Y.; writing—review and editing, Q.Y., J.J.; visualization, J.D. and J.J.; supervision, Q.Y. and J.D.; project administration, Q.Y.; funding acquisition, J.J. All authors have read and agreed to the published version of the manuscript.

Funding: This research was sponsored by Shanghai Gaofeng and the Gaoyuan Project for University Academic Program Development.

Conflicts of Interest: The authors declare no conflict of interest.

\section{References}

1. Schnapf, J.L.; Baylor, D.A. How photoreceptor cells respond to light. Sci. Am. 1987, 256, 40-47. [CrossRef]

2. Thapan, K.; Arendt, J.; Skene, D.J. An action spectrum for melatonin suppression: Evidence for a novel non-rod, non-cone photoreceptor system in humans. J. Physiol. 2001, 535, 261-267.

3. Brainard, G.C.; Hanifin, J.P.; Greeson, J.M.; Byrne, B.; Glickman, G.; Gerner, E.; Rollag, M. Action spectrum for melatonin regulation in humans: Evidence for a novel circadian photoreceptor. J. Neurosci. 2001, 21, 6405-6412. [CrossRef]

4. Rea, M.S.; Figueiro, M.G.; Bierman, A.; Bullough, J.D. Circadian light. J. Circadian Rhythms. 2010, 8, 2.

5. Lucas, R.J.; Peirson, S.N.; Berson, D.M.; Brown, T.M.; Cooper, H.M.; Czeisler, C.A.; Figueiro, M.G.; Gamlin, P.D.; Lockley, S.W.; Hagan, J.B.O.; et al. Measuring and using light in the melanopsin age. Trends Neurosci. 2014, 37, 1-9.

6. Glickman, G.; Levin, R.; Brainard, G.C. Ocular input for human melatonin regulation: Relevance to breast cancer. Neuroendocrinol. Lett. 2002, 23, 17-22.

7. Yao, Q.; Wang, H.B.; Uttley, J.; Zhuang, X.B. Illuminance reconstruction of road lighting in urban areas for efficient and healthy lighting performance evaluation. Appl. Sci. 2018, 8, 9.

8. Schreuder, D. Introduction: The function of outdoor lighting. In Outdoor Lighting: Physics, Vision and Perception; Springer: Dordrecht, The Netherlands, 2008.

9. Fotios, S.; Gibbons, R. Road lighting research for drivers and pedestrians: The basis of luminance and illuminance recommendations. Light. Res. Technol. 2018, 50, 154-186.

10. CIE 191:2010. Recommended System for Mesopic Photometry Based on Visual Performance; CIE Central Bureau Kegelgasse: Vienna, Austria, 2010.

11. Baldridge, A.M.; Hook, S.J.; Grove, C.I.; Rivera, G. The ASTER Spectral Library Version 2.0. Remote Sens. Environ. 2009, 113, 711-715.

12. Pan, Y.F.; Zhang, X.F.; Tian, J.; Jin, X.; Luo, L.; Yang, K. Mapping asphalt pavement aging and condition using multiple endmember spectral mixture analysis in Beijing, China. J. Appl. Remote Sens. 2017, 11, 016003.

13. Barlow, H.B. Purkinje shift and retinal noise. Nature 1957, 179, 255-256.

14. Li, H.C.; Sun, P.L.; Huang, Y.N.; Luo, M.R. Spectral Optimization of White LED based on Mesopic Luminance and Color Gamut Volume for Dim Lighting Conditions. Appl. Sci. 2020, 10, 3579. [CrossRef]

15. Zan, L.; Lin, D.; Zhong, P.; He, G. Optimal spectra of white LED integrated with quantum dots for mesopic vision. Opt. Express 2016, 24, 7643-7653.

16. Xiao, L.C.; Zhang, C.W.; Zhong, P.; He, G.X. Spectral optimization of phosphor-coated white LED for road lighting based on the mesopic limited luminous efficacy and IES color fidelity index. Appl. Opt. 2018, 57, 931-936.

17. Gibbons, R.B.; Terry, T.; Bhagavathula, R.; Meyer, J.; Lewis, A. Applicability of mesopic factors to the driving task. Light. Res. Technol. 2016, 48, 70-82.

18. Panda, S.; Nayak, S.K.; Campo, B.; Walker, J.R.; Hogenesch, J.B.; Jegla, T. Illumination of the melanopsin signaling pathway. Science 2005, 307, 600-604.

19. Qiu, X.D.; Kumbalasiri, T.; Carlson, S.M.; Wong, K.Y.; Krishna, V.; Provencio, I.; Berson, D.M. Induction of photosensitivity by heterologous expression of melanopsin. Nature 2005, 433, 745-749. 
20. Koyanagi, M.; Kubokawa, K.; Tsukamoto, H.; Shichida, Y.; Terakita, A. Cephalochordate melanopsin: Evolutionary linkage between invertebrate visual cells and vertebrate photosensitive retinal ganglion cells. Curr. Biol. 2005, 15, 1065-1069. [CrossRef]

21. Torii, M.; Kojima, D.; Okano, T.; Nakamura, A.; Terakita, A.; Shichida, Y.; Wada, A.; Fukada, Y. Two isoforms of chicken melanopsins show blue light sensitivity. FEBS Lett. 2007, 581, 5327-5331.

22. Bailes, H.J.; Lucas, R.J. Human melanopsin forms a pigment maximally sensitive to blue light $\left(\lambda_{\max } \approx 479 \mathrm{~nm}\right)$ supporting activation of Gq/11 and Gi/o signalling cascades. Proc. Biol. Sci. 2013, 280, 20122987.

23. Arendt, J. Melatonin and the pineal gland: Influence on mammalian seasonal and circadian physiology. Rev. Reprod. 1998, 3, 13-22. [CrossRef] [PubMed]

24. Reiter, R.J. Pineal gland: Interface between the photoperiodic environment and the endocrine system. Trends Endocrinol. Metab. 1991, 2, 13-19.

25. Wetterberg, L. Light and Biological Rhythms in Man; Elsevier: Amsterdam, The Netherlands, 2014.

26. Circadian Lighting Design | WELL Standard. Available online: https://standard.wellcertified.com/light/ circadian-lighting-design (accessed on 2 August 2020).

27. Rea, M.; Figueiro, M.; Bierman, A.; Hamner, R. Modelling the spectral sensitivity of the human circadian system. Light. Res. Technol. 2012, 44, 386-396.

28. Rea, M.S.; Figueiro, M.G. Light as a circadian stimulus for architectural lighting. Light. Res. Technol. 2018, 50, 497-510.

29. Figueiro, M.G.; Rea, M.S. Office lighting and personal light exposures in two seasons: Impact on sleep and mood. Light. Res. Technol. 2016, 48, 352-364.

30. Acosta, I.; Leslie, R.P.; Figueiro, M.G. Analysis of circadian stimulus allowed by daylighting in hospital rooms. Light. Res. Technol. 2017, 49, 49-61.

31. Dai, Q.; Huang, Y.; Hao, L.; Lin, Y.; Chen, K. Spatial and spectral illumination design for energy-efficient circadian lighting. Build. Environ. 2018, 146, 216-225.

32. Dai, Q.; Cai, W.; Shi, W.; Hao, L.; Wei, M. A proposed lighting-design space: Circadian effect versus visual illuminance. Build. Environ. 2017, 122, 287-293. [CrossRef]

33. Dai, Q.; Cai, W.; Hao, L.; Shi, W.; Wang, Z. Spectral optimisation and a novel lighting-design space based on circadian stimulus. Light. Res. Technol. 2018, 50, 1198-1211.

34. Dai, Q.; Shan, Q.F.; Lam, H.; Hao, L.; Lin, Y.; Cui, Z. Circadian-Effect engineering of solid-state lighting spectra for beneficial and tunable lighting. Opt. Express 2016, 24, 20049-20058.

35. Lewy, A.J.; Wehr, T.A.; Goodwin, F.K.; Newsome, D.A.; Markey, P. Light suppresses melatonin secretion in humans. Science 1980, 210, 1267-1269. [CrossRef] [PubMed]

36. Wright, K.P., Jr.; Hughes, R.J.; Kronauer, R.E.; Dijk, D.J.; Czeisler, C.A. Intrinsicnear-24-h pacemaker period determines limits of circadian entrainment to a weak synchronizer in humans. Proc. Natl. Acad. Sci. USA 2001, 98, 14027-14032.

37. Phillips, A.J.; Vidafar, P.; Burns, A.C.; McGlashan, E.M.; Anderson, C.; Rajaratnam, S.M.; Cain, S.W. High sensitivity and interindividual variability in the response of the human circadian system to evening light. Proc. Natl. Acad. Sci. USA 2019, 116, 12019-12024.

38. Rea, M.S.; Radetsky, L.C.; Bullough, J.D. Toward a model of outdoor lighting scene brightness. Light. Res. Technol. 2011, 43, 7-30.

39. Besenecker, U.C.; Bullough, J.D. Investigating visual mechanisms underlying scene brightness. Light. Res. Technol. 2017, 49, 16-32.

40. Brown, T.M.; Tsujimura, S.I.; Allen, A.E.; Wynne, J.; Bedford, R.; Vickery, G.; Vugler, A.; Lucas, R.J. Melanopsin-based brightness discrimination in mice and humans. Curr. Biol. 2012, 22, 1134-1141. [PubMed]

41. Falchi, F.; Cinzano, P.; Elvidge, C.D. Limiting the impact of light pollution on human health, environment and stellar visibility. J. Environ. Manag. 2011, 92, 2714-2722.

42. Hollan, J. Metabolism-Influencing light: Measurement by digital cameras. In Proceedings of the Cancer and Rhythm, Graz, Austria, 14-16 October 2004.

43. Illuminating Engineering Society. IES Method for Evaluating Light Source Colour Rendition; IES: New York, NY, USA, 2015; pp. 15-30. 
44. Rea, M.; Smith, A.; Bierman, A.; Figueiro, M.G. The Potential of Outdoor Lighting for Stimulating the Human Circadian System; Technical Report; Alliance for Solid-State Illumination Systems and Technologies (ASSIST): Troy, NY, USA, 2010.

45. Bullough, J.D.; Radetsky, L.C.; Besenecker, U.C.; Rea, M.S. Influence of Spectral Power Distribution on Scene Brightness at Different Light Levels. LEUKOS 2014, 10, 3-9. 